\title{
Some Preliminary Remarks on the Cenoscopic and Idioscopic aspects of Information Retrieval
}

\author{
Sachi Arafat \\ King Abdulaziz University \\ Saudi Arabia
}

\begin{abstract}
Human-computer information retrieval (HCIR) or IR is discipline/discourse and perhaps amongst the most commonly used applications-search-enabling our ubiquitous interconnected and technology-mediated modern reality. Technical discourses of IR aim to develop algorithms to effectively join users to information (documents, images, or more generally, information experiences). It also aims to evaluate its algorithms and systems, and to understand what works and what doesn't. This focus on discovering and analysing new phenomena pertaining to search strategies (and recommendation and ranking functions), user-interaction strategies and so on refers to the idioscopic dimension of IR. However, this discourses presumes-i.e. is founded-upon-deeper ideas pertaining for example to human-communication and psychology, problems of knowledge and about the structure of social reality, these are its cenoscopic or more philosophical aspects. This paper analyses some of these deeper ideas and how they have appeared in their corresponding idioscopic form as mathematical and cybernetic models of agent communication. This paper indicates that it is necessary to separate the idioscopic and cenoscopic aspects of such discourses to allow the respective phenomena to be analyzed according to the methodology proper to it.
\end{abstract}

\section{General Terms}

Information retrieval, Explainability, Eoundations

\section{Keywords}

Information retrieval, Edioscopic and cenoscopic, Problem of knowledge, Semantic gap, Explainability

\section{INTRODUCTION}

In the last two decades Information Retrieval (IR), that is, humancomputer information retrieval (HCIR) or search, has been understood to be an interaction between a user and the interface elements of a textbox and search button. By extension it has also been understood as including the following browsing processes. These activities overall resemble a communicative process between two or more human beings. More specifically, it resembles the humancommunicative process of question answering which we, following [5], will call human-human information retrieval, denoting by it a process as similar in simplicity to the HCIR. The modern trend of search-enabled ubiquitous devices, as [8, Chapter 1] explain, sig- nificantly change this communicate experience, such that the specific, traditional IR function, is no longer a good representative of that experience. Instead, as they argue, the experience is better understood in the general sense as a technology-mediated experience, and formally studied as such. This is a claim pertaining to the foundations of Information Retrieval and Science (IR\&S), and as such it pertains to the cenoscopic aspect of IR\&S not its idioscopic one in the terminology used by Peirce following Bentham [16].

The purpose of this paper is to contribute to the cenoscopic understanding of IR\&S developed in [8] by bringing to light some of the foundational issues apparent in the largely idioscopic exploration in [5] focusing on the methodology to discover and capitalize on new phenomena pertaining to search. Section 2 elucidates the dual problem of knowledge at the heart of both HCIR made complicated since the agents participating in the respective communication are of different kinds. It argues that in addition to linking to the classical problem of knowledge it also refers to the same in traditional cybernetics. Section 3 explains in what way this two-agent communication is founded on a social reality and is purposed to address the 'social gap' that is increased in some respect due to technological automation but is remedied in other respects by the same. This section also focuses on the particular educational context within that social reality, and how IR\&S enables a specific modality of it. Section 4 concludes that the concept of search given the modern context of ubiquitous systems as implied in [5] and further developed in $[\overline{8}]$, appears to be a primitive concept pertaining our inclination to know, now by means of technology, that is now a common to all particular areas of knowledge and not specific to IR\&S even though the latter studies it in a special capacity. Section 5 interpret the aspects understood as problems of knowledge in section 2 as problems of explainability, that is, explainable to users, researchers and 'explainable' to systems, and relates this to the recent trends in AI and explainability. Section 6 explains how the centrality of ostensive retrieval for idioscopic purposes in [5] is relevant in the context of ubiquitous systems with encapsulated search functions as explored in [8]. Finally section 7 concludes the paper.

\section{THE DUAL PROBLEMS OF KNOWLEDGE IN IR: USER AND SYSTEM UNDERSTANDING}

Compared to HHIR where participants use multiple senses, a complex language and possess advanced reasoning systems, HCIR is not only primitive but unfamiliar. key component of humancommunication is empathy, is understanding the otheranother hu- 
manin light of self-understanding. When the other is machine, the involvement of empathy, corresponding to an arrangement of psychological processes in the user, does not function in any typical manner. Similarly, analogically speaking, it is unfamiliar to the computer and to the designer of the search system. Not unfamiliar to himself directly as a human, but in the context of his job of creating operative models of users that will condition the responses of such a system. While in the HHIR case the participants are constantly figuring out what the others linguistic and non-linguistic expressions mean, in the HCIR case, both participants lack equivalent amount of access to this understanding.

Certainly, for the computer system, there is no understanding of meaning [8]: the nominalist or symbolic interreferencing between representations of user behavior are not understood by the system as they are for the human agent. The two agents are starkly different in kind. In order to better understand this difference [5] suggests recognizing the asymmetricity of it. This difference is not the typical difference there is in human-communication or HHIR more specifically, to which the problem of empathy refers. Instead, as the agents are actually different beings, the way one comes to know the other is unlike the way other does. Thus, there are two problems of knowledge or knowing in question, the way the system comes to know the user and the way the user comes to know the system, the system-understanding problem (SUP) and the user understanding problem (UUP) [5]. We think these are significant problems since (a) HCIR is predicated upon the processes of user and system understanding, (b) effective/valuable/beneficial HCIR corresponds to their effective resolution.

These two problems are actually different in kind as the agents doing the knowing are different in kind. The UUP refers to a process of learning on behalf of a machine and exhibiting machinebehavior in response guided by an algorithm. This is not strictly a knowing, or even any sort of real cogitative process, except in an analogical, simulated and/or metaphorical sense; and this is arguably the sense in which cognitive relates to human cognition in the cognitive sciences based on computational paradigms.

Given this difference in kind between the problems, it is clear that while non-trivial similarities exist in their character (in an analogical sense), the subject-matter of the problems belong to different sciences. The SUP is a problem primarily of psychologymeant in the traditional sense (i.e. relating to the entire human being not only behaviour, consciousness or purely mental aspects individually). And in this sense, it draws from epistemology and metaphysics, biology, and the practical sciences (such as politics and ethics). In the modern sense, while it could still primarily be categorized as a problem of psychology, such an assignment would entail some equivocation unlike when linking to traditional psychology since there are several often unrelated psychologies and other human and social sciences. Thus, the problem would be split amongst them in a manner that is as unclear as the current interrelations between these modern sciences and their corresponding discourses.

The UUP would traditionally be a problem of mathematics, the arts and crafts. In the modern time it is a problem of mathematical computation and computational-paradigm inspired cognitive science. This is already well-realized in the current IR discourse. As they are problems in different sciences, both modern and traditional, one expects their corresponding expressionsbased on the respective terminology of their sciencesas well as the methodology for their solution, that is for effective/successful to differ, perhaps considerably.

The approach of [5] addresses this difference using the computational-cognitive science perspective, by analogically un- derstanding the user to be a system, so that both the SUP and UUP can be understood as a problem pertaining to the sciences appropriate to handle the latter: the computational sciences. However, it does this while explicitly acknowledging the much-reduced character of this abstraction. In particular, [5] models HCIR as a series of processes involving two systems (which can be understood also as one bi-stable system: interpretation/expression, observation and internal processes, see 1 The changes or dynamics of this system are defined by a sequence of such processes and a set of corresponding states that are affected thereby; and in this sense this is an abstract state model. There is no restriction as to how detailed this can get. Each such process can be expressed in terms of arbitrarily deeply nested sub-processes of such types (or sub-types), and hence concurrent sub-events. This is therefore a fully atomistic model where each level of depth exhibits a rationality. For example, at one level you have bi-stable system with state changes, at a more refined level there is a representations of psychological state changes as internal processes within the user entity/system (the image on the right of 11) and so on.

What makes this specifically a bistable system-in the sense of cybernetics [10, 36, 37]- instead of a more general twopart system is that there are goals whose achievement results in a favorable or stable overall state; the movement/dynamics is purposeful. Or in the classical sense, there is a final cause to this overall system [27 20, 31]. This goal is defined for the overall (bistable) system as the information need (IN), the notion common to IR discourse. Moreover, at the level of each entity, there is a breakdown of the functions, or in a classical sense, powers/abilities/faculties, constituting them. Thus, the user and system (analogically of course) is able to reason, remember, be-present (session layer, see 2), actintentionally through gestures (gesture layer) or elicit not-quite as meaningful (as gestures) movements (physical layer). Each cue observed by one entity from the other is progressively assigned layers of meaning as it travels through the corresponding faculties.

As [5] also explains however, this atomistic bistable system, while expressing the relation between the two relevant entities in HCIR, does not capture what happens in the investigation of a HCIR, where necessarily an observer or researcher is involved. Instead, the researcher or observer entity must also be understood to be part of the overall model for capturing the UUP/SUP problems. This was influenced mainly from the situation of quantum theory [35, 4, 30, 6, 38, 3, 17]. There are several important particulars of the model that are similarly influenced from quantum theory. In fact, that it is an arbitrarily atomistic abstract state system (where a state is simply a collection of knowable properties) structured into 'knowables able to be known by the other entity or the researcher, is directly influenced by the way the operator-theoretical wavefunction is used to capture the state of a physical system and the way (and properties) of knowing that state (through measuring apparatus). Further details of this is in [5] and a forthcoming work.

While such a model is indeed limited, since it reduces the human dimensions of the SUP to dead/dry computational aspects, it does fulfill an important function. That is, it provides a computational-mathematical perspective of the problem, something that will regardless be required to link any future more humanistic understanding of the SUP to the necessarily mathematicalcomputational framing of the UUP. The work in [8] undertakes the task of developing this more humanistic understanding. 


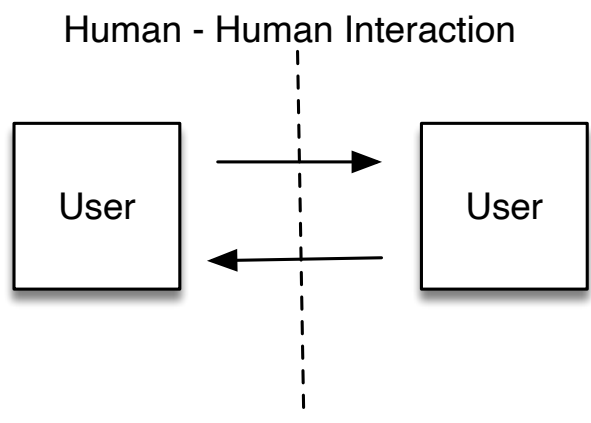

Proxy-Proxy Communication Or Indirect human communication

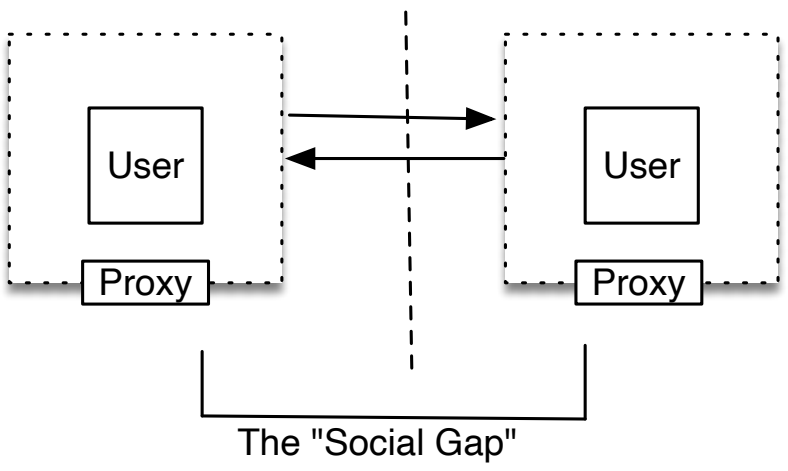

"Proxified" discursive tradition based societies
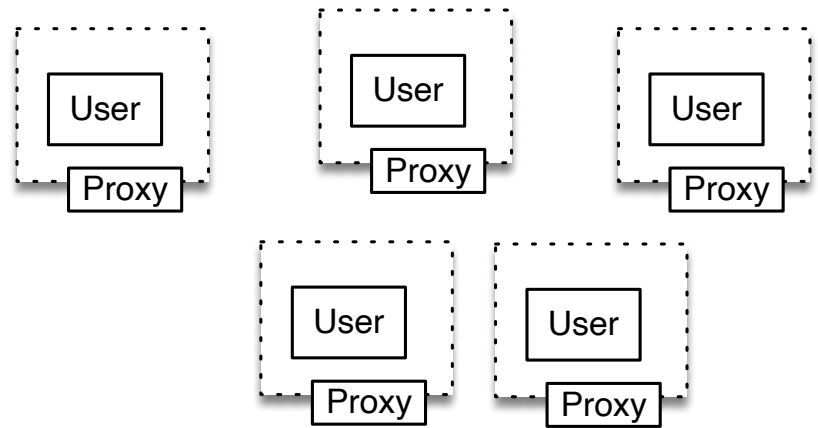

Fig. 1. HHIR as proxy-based communication founded on social gaps

\section{THE INSTRINSIC SOCIAL DIMENSION OF HCIR AND THE GENERALIZATION OF THE SEMANTIC GAP}

While the above bistable model captures something of the interaction events that constitute HCIR, it fails to capture the social dimension. This is of course not simply a matter of increasing the number of entities within such a model. HCIR systems do in fact have social roles, and to see what this is, we need to see the social function

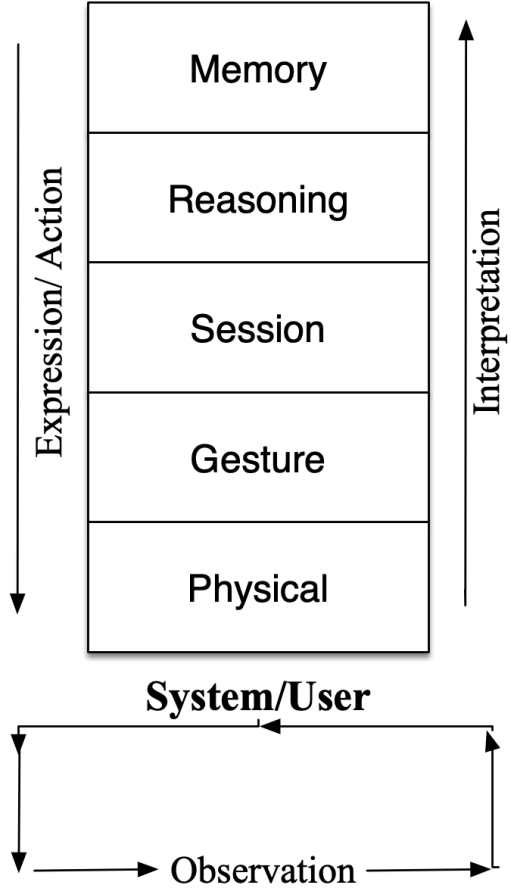

Fig. 2. HHIR as a bistable system constituted by several types of processes and multi-aspect agent systems

of HHIR, thus HHIR needs to be understood from a sociological perspective. HCIR (searching) takes on a special sociological role in current times (see Arafat and Ashoori 2019) and encourages a particular social perspective in a searcher searching over long periods of time. This perspective is roughly speaking the sociological imagination (Mills, 1959).

In the modern age, the automation of services through the use of technology, both from a top-down and bottom-up sense [8. Chapter 1], has replaced numerous scenarios of human-human interaction. Human communication in such scenarios, and social (interpersonal communication) scenarios evolved into ones involving devices acting as proxies to human agents. This can be understood as introducing a social gap between people by replacing the social experience with a real other, with a technology-mediated experience of the other [8]. An experience that involves dealing with abstract representations of the other. While this still allows similar cognitive processes, directed at the other, to happen, the mediated experience of the other is potentially of a different kind.

Social gaps are not only manifest when social scenarios are augmented with proxies. Indeed, the lack of similarity in the knowledge and understanding of any two agents means a gap in understanding/knowledge, or even language or any other attribute of an agent. Thus, there is always a gap in some sense, and one could argue that these sorts of gaps limit the communicative presence of agents and hence are effectually like talking to a reduced version of an other. We would argue that while communicative-presence is indeed reduced in these senses, the scenario of not having the other present is of a different kind. There is a long discussion to be had about the different levels and categories of communicative presence [19, 32, 33], and we will leave this for a future occasion. 
Of course social gaps are inevitable for automation, and automation is one of the main purposes of technology. Moreover, while automation leads to social gaps in one context it could close gaps in other areas, creating new opportunities for communicationwhether through proxies or otherwisethat previously did not exist; social networking is the typical example of this. Social gaps could be reduced in a more indirect sense due to automation and the corresponding mediation of technology, such as through enabling opportunities for interaction between persons and knowledge entities that are already proxies of persons: the written word (books, academic papers, other writings), multimedia, etc. This is the whole purpose of HCIR systems. One has to differentiate however between such dead proxy objects and the interaction with live persons through a proxy or otherwise: factual information, or the learning of facts vastly underdetermines social interaction. Instead the latter facilitates human experiences as different as the development of identity, to psychological therapy, to the experience of care and mutual-empathy.

Why is this all relevant? Our main point here is to understand the social gap as a background to several notions used in IR discourse, the most important of them being the semantic gap referring to the difference between the form of a retrieved object (such as the document or a part thereof) and its meaning. This gap includes of course that which is the literal (or commonly understood) meaning of a word (or a set of words) and any other meanings. However, in the context of HCIR it also has an additional component. In a normal communicative scenario between two persons any gap in the understanding of expressions in some part of the process can potentially be resolved through further conversation, in an explicit manner. In the context of proxies, the nature of this gap is modified. In particular, there are a more limited set of possibilities.

Moreover, this semantic gap is not only a static fact about (a) words and their meanings in general, or the (b) limiting of possibilities of understanding meanings of expressions due to the social gap, but an attribute of a live search scenario, an informational experience [5], what [8] construes more generally as a technologymediated experience (TME). As an attribute, and furthermore, a constituent of that experience, it conditions and is conditioned by what [8] called the efficient cause of the event of a TME, the information need, which is usually taken in HCIR discourses to be an attribute of a user. The fulfillment of needs through such experiences are limited by the existence of gaps whether they be permanent or temporary, and in this sense gaps are limits to what users can know. They ultimately pertain to the problem of knowledge as discussed in the prior section; not specifically to the user/system understanding problems, but the general problem of knowing through devices/instruments; thus gaps are epistemic limits due to the ontological nature of search scenarios. Our point is that these epistemic limits, or more accurately, social-epistemic limits, are natural to our technology-mediated society. Thus, the HCIR as a substitute of HHIR, engenders a social experience that is strictly limited in various ways even though it creates new opportunities for social experience.

The sociological imagination [26, 9], the lucid awareness (or being-in-the-world/society) of the relationship between personal experience and the wider society, is the tacit background cogitation, in light of one's private identity (personas) with respect to its reflection in ones understanding of the public sphere, that informs our daily activities. Our living in the modern world, through the use of ubiquitous devices form an understanding of this public sphere, and also inform how we develop our private identities. Our expressions in this public sphere (e.g. Tweets) is our avatar that plays out in this proxy world. Our sociological imagination is then in terms of our expressions in this public space, and the internalization of this public sphere in us. Users are concerned about public issues through their proxy representations, through media, i.e. arbitrarily mediated forms. Our information needs are based on already technology-mediated perceptions of the world. Thus, to understand an information need and the form it takes in user action (queries)as would be required for any scientific IR seeking to explain needs and queriesmeans to go beyond the individual to investigate how social structures shape individuals and their action. It means to understand how the social gap limits the sociological imagination.

As technological-mediation becomes increasingly our normative mode of living, what is our proxy-life (represented through our avatars) become more and more central to our selfunderstanding. The extent depends on the relationship between the proxy and the person represented, and also in general between man and machine. Understanding the extent to which user are their proxies are important to resolve the user-understanding problem (UUP). The UUP similarly cannot be effectively resolved without understanding the social gap that is the foundation of their information need and hence information experience; and this requires analysis of the purposes of automation that lead to the proxy substitutions engendering the gap in the first place. That is, why did the user engage with the IR system in the first place, why did they leave the types of proxy information that they did and what kinds of IN can be inferred from it. The proxy information here is not just the query but any arbitrary details about the user.

In addressing the question of why they engaged the system in the first place, the most general answer is that the nature of users as persons is to know, that they seek meaning, and IR systems are therefore means to meaning-suggestion (or meaning-fulfilment) [8. Chapter 5]. This general answer can be made more specific by noticing that the existence of gaps implies the tendency to bridge them. Thus, the action of requesting information from a system is some aspect of socialization, or more generally it is an activity pertaining to not only to the fulfilment of a specific task but also a consequence of their being-in-the-world. HCIR, to the extent that there is a social gap and hence a natural tendency to address it, is a social activity (even if there are no additional human users involved). HCIR is then a means of and furthermore, induces, sociological imagination.

\subsection{THE SPECIFIC RELATIONSHIP BETWEEN SEARCH AND EDUCATION}

In terms of human-human interaction, education in the modern sense can be understood as a set of processes involving institutions (a set of proxies/people) and individual (proxies/people). The institution of education started locally in individual homes, then moved to the local community in the form of a school, later still an interesting phenomena became visible among many institutions as characterized by assembly line production strategy: the use of humans in an industrial process in the interest of 'automation' began to resemble the organizational structure of a machine-only process. The mass-production strategy influenced the education establishment; yet, although there is a reduced connection between individuals (as emphasized by the social gap) it certainly exists at the least within the bounds of social protocol within an institution (an example of social protocol is professionalism at work). This connection is most apparent between students and their educators where it is assumed that the HHIR in the educational context between academics represents the most effective or optimal search. In particular the educational context that is to be modelled for investigation of HHIR has changed over the years by integrating automated components. 
The idea of proxies for education is not new, an educational culture based on automated, and 'ad-hoc' learning that is a reality in our time, was conceptualized as an 'educational web' in (Illich, 1971), see [7, 14, 24, 13] for more details. Educational webs enable moments of living to be transformed into moments of learning (Illich, 1971). This is particularly the case where the moments of living are technology-mediated living. Illich sees such as webs as reducing social gaps not due to automation as such but social inequities.

\section{SEARCH AS PRIMITIVE}

If we take search thereby as the natural tendency to address gaps, which are most generally gaps in meaning (and hence we look for meaning-suggestion), and more specifically semantic/social and other gaps, then search can be understood as a primitive. The work in [5] takes it as such, that is, it doesnt seek to further define it in terms of other concepts. Thus, search as embodied in tools, functions (mathematical, programmatic, social, etc.), and as an activity which is a means of technology-mediated living. Search as the use of IR systems is then a proxy that brokers interactions between a user and other proxies. Search both forms and reflects the habitus of its users, it forms and is used to reflect upon their sociological imagination. This is apparent once it is not perceived as an isolated, minor activity of computer use, but somehow, all of technology-use. Search is not simply the use of simple web-based tools, but the interaction with documents, the selecting of programs, the saving documents or any activity that indeed results in any type of meaning-fulfilment. The user in modern society is then searching for what in hindsight would be their future identities or future selves represented by 'interaction proxies' that contain instances of general computer usage. These are their interactions with social media, and other interaction with anything artificial, any other TME that effect their understanding.

Search in this sense is the impetus for interaction and socialization presupposed by HCIR/HHIR and not only engendered by them. As such, search is not primarily a technical subject area relevant for HCIR/HHIR but for the wider human sciences in general.

\section{SYSTEM AND USER UNDERSTANDING PROBLEMS AS AS PROBLEMS OF EXPLAINABILITY AND INTELLIGIBILITY}

The process of computational search corresponds to a cogitative and active process on behalf of the user, and a response on behalf of the system upon associating data entities relevant to the users actions. The traditional problem of query formulation (QFP) pertains to the difficulty on the users part in deciding and formulating what they are actually searching for. Not only is this a difficult task, since their IN is often vague, but it is changing throughout search. Were the IN to be well defined, most users would still struggle to comprehend the relationship between their query and the system's response. This lack of knowhow about the retrieval system limits the efficiency and effectiveness of search. This is why the SUP is an important problem, but it remains little highlighted as a distinct and important problem in the literature. Any resolution to this problem will inform the user interface and the entire user-interaction strategy of the system. The QFP in turn relates to the UUP as a system always needs to ask how the query it is given represents the IN.

To resolve the SUP does not mean to transform normal users to experts or researchers who know the details of algorithms, instead this is a problem about explainability and intelligibility as is commonly discussed in explainable AI discourse [2, 18, 25, 1].
Instead, it is about enabling informational experiences resembling HHIR scenarios where appropriate. In the HHIR context the discussants are familiar with one another as human agents, there is an already existent and deep intelligibility of expressions and behavior; there is much already explained, consistent and coherent. Typical IR/HCIR systems, and now, AI systems in general where explainability is important, are very artificial in comparison as human interpretable explanations are not important therein; they remain largely black-boxes. What however is the ideal form of HCIR experience? Should some HHIR scenario be the standard for every corresponding HCIR scenario? In HHIR, each agent conceptualizes the other in terms of psychological ideas such as personality, habit or nature, emotions or affect, etc., the content of such concepts contribute to their overall understanding of the other and to the way a question-answering scenario proceed; compare this to the a much reduced scenario in HCIR which is problematized by the SUP and UUP.

From the perspective of the system the UUP is about the explainability of user behavior. Whereas explainability for an artificial system may only be to relate between variables in order to respond effectively to a user query, it is explainability in its full sense for the researcher who uses the data captured on such a system to understand the HCIR scenario. Thus, the user seeks explanations of system behavior, the researcher and system that of user behavior but in different senses. Seeking explanation involves first and foremost the seeking out of the intelligible in any such HCIR scenario, i.e. seeking out meaningful elements in the expression of the other. For the researcher this means developing a methodology through which to categorize and make sense of user behavior with respect to what they point to as to the psychological intentions and mental action, and what can be inferred from them as to stable (natures or habits, such as persona) or unstable properties (such as affects). This presupposes a philosophical (cenoscopic, to use a Peircean term) psychology as well as corresponding empirical (idioscopic) psychologies [29 28]. Where idioscopic psychology here would include models of cognition in the cognitive sciences such as [21, 22, 23, 15].

For the system, the corresponding methodology for resolving the UUP would need to come from a reductive idioscopic psychology that nevertheless is based on a cenoscopic psychology on which researchers base their investigation. The work in [5] suggested quantum theory as such a reductive idioscopic base without developing any corresponding cenoscopic foundation while [8] can be understood as heading in this direction. Since the SUP/UUP are ultimately problems of knowledge, their resolution must begin at the cenoscopic level. However, to the extent that we want to resolve these problems for real instances of technology-mediated experience, they have a contextual-empirical aspect, they are not merely theoretical problems but have practical import for users, and so there requires to be an idioscopic science (an empirical psychology) that deals directly with the data of user interaction.

\section{THE IMPORTANCE OF THE OSTENSIVE INFORMATION RETRIEVAL MODEL IN THE CONTEXT OF UBIQUITIOUS SYSTEMS}

The idea of the ostensive information retrieval (OIR) paradigm of $[12,11,34]$ is particularly important to better understand both the UUP and SUP, not that this was explicitly intended by its author. Originally the OIR was developed as a way to address the ambiguous and changing information needs in users, those that we except them to express through formulating queries. The idea was that instead of asking them to formulate queries they could instead indi- 
cate what they sought without forming words. The user interface would require to be such that the entire HCIR journey, the initial search (which might require words) and the browsing that followed would be displayed in a form that helps users disambiguate their information needs. That is, it follows the user through several states of knowledge. Moreover, the interface and underlying algorithm treated newer interactions as more important than older interactions as a way to accommodate changing needs. While a practically useful model, its main use we think lay in the cenoscopic questions it raises which it answers in an ad-hoc fashion at the idioscopic level: what is cogitation, and what is going through the mind of the user now that is relevant. While philosophical psychology has much to say about the former, the latter question poses a difficulty in that no amount of analysis of neural/synaptic correlates of cogitation will reveal what is actually thought. In fact, the problem of what can or cannot be known about mind through its bodily correlates (which synapses are) goes to the heart of the mind-body problem and others in the philosophy of mind.

Allowing the user to communicate by pointing than forming words on some level means a less-reductive expression to the extent there is a reduction of meaning from concepts to words. Yet, pointing to existing interface elements is less expressive than words for scenarios where the user has a way to express their need in words and where the available objects for pointing are less expressive or exact. The idea is that whatever is lost due to lack of specificity of interface elements over words can be made up through the specification that necessarily happens in the subsequent browsing process so that the user is homing-in onto a specific answer. Consider the expanded version of this notion of OIR for our current sociotechnical reality of the ubiquity of devices as developed in $[8]$. Let the browsing process involve the entirety of a users living in the world through technology, such as they are not merely pointing to items on screen but doing a sequence of tasks (e.g. walking to a location, taking a picture then chatting to a friend), and the systemtaking their cue from the entirety of lived-experiencesupports/responds in a way as to make this experience better. We consider this to be the natural extension of the idea of OIR for the modern time, and it better sets the context for UUP/SUP. Thus, it is not just that UUP/SUP is about two-agent interaction understood as a bistable system, but the living-in-theworld of one agent and the natural coupling/entanglement of the second agent (the system) in a way as to help that that or to improve their experience somehow. Seen in this way, the UUP/SUP take on a further ethical dimension that is not as prominent when seen in the context of simple interaction (or from the perspective at the interactive layer as discussed in Arafat and Ashoori 2019, Chapter 5).

In any such generalized OIR type strategy enabled by systems engaging in HHIR, the SUP has to do with understanding the language of ostension. To the extent the goal of such a strategy is to keep out of the users way, this language in primarily a non-linguistic sign language, and only secondarily one involving natural language. The scientific development of any such language (i.e. user-interfaces and user-interaction strategies) needs recourse to the cenoscopic and idioscopic bases discussed above.

\section{CONCLUSIONS}

As the arguments above indicate it is not only useful but important to separate out the cenoscopic (philosophical and foundational aspects, roughly speaking) and idioscopic (the phenomenal and empirical) aspects of a modern discourse such as human-computer information retrieval (HCIR) (or IR as it is more commonly known).
This is so that the respective problems therein can be correctly identified as investigated according to methods proper to it. This paper brought out some salient problems of the subject area studied by IR. It identified foundational problems, which are not directly studied by HCIR but instead positions regarding them are presumed. They would therefore belong to the cenoscopic aspect of IR not the idioscopic aspect of the larger IR\&S discourse. Upon [8] studying the kinds of questions this cenoscopic aspect would investigate, it was possible to identity several issues posed in the mainly idioscopic study of [8] as cenoscopic problems.

As the above discussed these are the (a) user and system understanding problems, previously seen as an (idioscopic) a problem of bistable system stability in the context of cybernetics, but now as (i) problems of knowledge (i.e. epistemology, classical psychology etc.) as well as (ii) mutual explainability and intelligibility, (b) the hierarchy of 'gaps' (social, semantic etc.), previously identified as the justification for uncertainty represented by probability in idioscopic investigations, now identified as facts of social reality, (c) the importance of IR for education previously noted only as an important 'application' of search but now seen as enabling as a spontaneous educational culture (i.e. effecting social reality in a specific, sustained way), (d) search as a primitive practical aspect of all knowing regardless of discipline, as a 'primitive' attribute of knowing in the modern time, and not just an 'application'/software and finally (e) ostensive information retrieval as not just a model of IR as in the idiographic case but as capturing what the modern, encapsulated search (meaning, as hidden amongst other applications or device-use in general) is about from the perspective of a philosophy of technology.

\section{REFERENCES}

[1] Ashraf Abdul, Jo Vermeulen, Danding Wang, Brian Y Lim, and Mohan Kankanhalli. Trends and trajectories for explainable, accountable and intelligible systems: An hci research agenda. In Proceedings of the $2018 \mathrm{CHI}$ conference on human factors in computing systems, page 582. ACM, 2018.

[2] Amina Adadi and Mohammed Berrada. Peeking inside the black-box: A survey on explainable artificial intelligence (xai). IEEE Access, 6:52138-52160, 2018.

[3] Diederik Aerts, Jonito Aerts Arguëlles, Lester Beltran, Lyneth Beltran, Isaac Distrito, Massimiliano Sassoli de Bianchi, Sandro Sozzo, and Tomas Veloz. Towards a quantum world wide web. Theoretical Computer Science, 752:116-131, 2018.

[4] S. Arafat and C. J. van Rijsbergen. Quantum theory and the nature of search. In Proceedings of the AAAI Symposium on Quantum Interaction, pages 114-121, 2007.

[5] Sachi Arafat. Foundations research in information retrieval inspired by quantum theory. $\mathrm{PhD}$ thesis, University of Glasgow, 2008.

[6] Sachi Arafat. Senses in which quantum theory is an analogy for information retrieval and science. In Dawei Song, Massimo Melucci, Ingo Frommholz, Peng Zhang, Lei Wang, and Sachi Arafat, editors, Quantum Interaction, pages 161-171, Berlin, Heidelberg, 2011. Springer Berlin Heidelberg.

[7] Sachi Arafat, Naif Aljohani, Rabeeh Abbasi, Amir Hussain, and Miltiadis Lytras. Connections between e-learning, web science, cognitive computation and social sensing, and their relevance to learning analytics: A preliminary study. Computers in Human Behavior, 92:478 - 486, 2019. 
[8] Sachi Arafat and Elham Ashoori. Search Foundations: Towards a Science of Technology-mediated Experience. MIT Press, 2019.

[9] Stanley Aronowitz. A mills revival. Logos, 2(3):67-93, 2003.

[10] W Ross Ashby. An introduction to cybernetics. Chapman \& Hall Ltd, 1961.

[11] I. Campbell. The Ostensive Model of Developing Information Needs. Ph.D. dissertation, Department of Computer Science, University of Glasgow, 2000.

[12] I. Campbell and C. J. van Rijsbergen. The ostensive model of developing information needs. CoLIS, page 251268, 1996.

[13] Teresa J Carter and Bryan Adkins. Situated learning, communities of practice, and the social construction of knowledge. Theory and Practice of Adult and Higher Education, page $113,2017$.

[14] Lisa Dawley and Chris Dede. Situated learning in virtual worlds and immersive simulations. In Handbook of research on educational communications and technology, pages 723734. Springer, 2014.

[15] M. De Mey. The cognitive paradigm.

[16] John Deely. Four ages of understanding: The first postmodern survey of philosophy from ancient times to the turn of the twenty-first century. University of Toronto Press, 2001.

[17] Emanuele Di Buccio and Massimo Melucci. Searching for information with meet and join operators. In QuantumLike Models for Information Retrieval and Decision-Making, pages 145-168. Springer, 2019.

[18] Filip Karlo Došilović, Mario Brčić, and Nikica Hlupić. Explainable artificial intelligence: A survey. In 2018 41st International convention on information and communication technology, electronics and microelectronics (MIPRO), pages 0210-0215. IEEE, 2018.

[19] Hubert L Dreyfus. On the internet. Routledge, 2013.

[20] Edward Feser. Scholastic metaphysics: A contemporary introduction, volume 39. Editiones Scholasticae Heusenstamm, 2014.

[21] Peter Gärdenfors. Knowledge in flux: Modeling the dynamics of epistemic states. The MIT Press, 1988.

[22] Peter Gärdenfors. Conceptual spaces: The geometry of thought. MIT press, 2004.

[23] Peter Gärdenfors. The Geometry of Meaning: Semantics Based on Conceptual Spaces. MIT Press, 2014.

[24] James Hendler and Alice Mulvehill. Social Machines: The Coming Collision of Artificial Intelligence, Social Networking, and Humanity. Apress, 2016.

[25] Tim Miller. Explanation in artificial intelligence: Insights from the social sciences. Artificial Intelligence, 2018.

[26] C Wright Mills. The sociological imagination. Oxford University Press, 2000.

[27] Martha C Nussbaum and Amélie Oksenberg Rorty. Essays on Aristotle's De anima. Clarendon Press, 1992.

[28] Charles S Peirce. The essential peirce: Selected philosophical writings, vol. 2, peirce edition project. Bloomington/Indianapolis, 1998.

[29] Charles Sanders Peirce. The Essential Peirce, Volume 1: Selected Philosophical Writings (1867-1893), volume 1. Indiana University Press, 1992.
[30] D. Song, M. Lalmas, CJ van Rijsbergen, I. Frommholz, B. Piwowarski, J. Wang, P. Zhang, G. Zuccon, PD Bruza, S. Arafat, et al. How quantum theory is developing the field of information retrieval. In 2010 AAAI Fall Symposium Series, 2010.

[31] Richard Sorabji. Aristotle on memory. University Of Chicago Press, 2 edition, 2006.

[32] Sherry Turkle. The second self: Computers and the human spirit. Mit Press, 2005.

[33] Sherry Turkle. Life on the Screen. Simon and Schuster, 2011.

[34] C. J. van Rijsbergen. Quantum logic and information retrieval. In 2nd Workshop on Information Retrieval, Uncertainty and Logic, IR Group, University of Glasgow, Scotland, 1996.

[35] C. J. van Rijsbergen. The Geometry Of Information Retrieval. Cambridge University Press, 2004.

[36] Heinz Von Foerster. Understanding understanding: Essays on cybernetics and cognition. Springer Science \& Business Media, 2007.

[37] Norbert Wiener. The human use of human beings: Cybernetics and society. Number 320. Da Capo Press, 1988.

[38] Peng Zhang, Zhan Su, Lipeng Zhang, Benyou Wang, and Dawei Song. A quantum many-body wave function inspired language modeling approach. In Proceedings of the 27th ACM International Conference on Information and Knowledge Management, pages 1303-1312. ACM, 2018. 